\title{
Syphilitic Meningitis in HIV-Patients With Meningeal Syndrome: Report of Two Cases and Review
}

\author{
Ricardo Andrade Carmo, Alexandre Sampaio Moura, \\ Paulo Pereira Christo, Ana Cláudia Morandi and \\ Marcelo Silva Oliveira
}

Eduardo de Menezes Hospital and Federal University of Minas Gerais, Belo Horizonte, MG, Brazil

Few patients with symptomatic neurosyphilis present with signs and symptoms of acute meningitis. Here we report two cases of syphilitic meningitis diagnosed in HIV patients with meningeal syndrome. The first case, a 30-year-old black bisexual male, had concurrent meningeal and ocular syphilis with persistent unusually low CSF glucose levels. He responded well to 21 days of intravenous penicillin therapy. The second case was a 55-year-old female with epilepsy, depression, behavioral disorder and confusion. The diagnosis of HIV infection was made after onset of the syphilitic meningitis. She was treated with 21 days i.v. penicillin with improvement in her clinical condition. The clinical aspects of combined neurosyphilis and HIV infection, plus special features of diagnosis and treatment are discussed.

Key Words: AIDS, meningitis, neurosyphilis.

Despite great improvements in diagnosis and treatment observed throughout the 20th century, syphilis still poses a challenge it is responsible for significant morbidity in a number of different regions of the world $[1,2]$. The close association of syphilis with the human immunodeficiency virus (HIV) has been frequently reported and is of growing public health concern in developed and developing countries [3, 4].

Historically, symptomatic neurosyphilis developed in $4 \%$ to $9 \%$ of patients with untreated syphilis $[5,6]$. It was diagnosed in $10 \%$ of patients with latent syphilis presenting with acute psychiatric illness [7] and, in 12\%, presenting with neurologic illness [8].

Received on 6 May 2001; revised 11 October 2001.

Address for correspondence: Dr. Ricardo A. Carmo, MD. Eduardo de Menezes Hospital.Rua Dr. Cristiano Resende, 2213. Belo Horizonte, MG. Zip Code: 30622-020, Brazil. Phone: + 55 313383 8000. Fax: + 553133838527.

E-mail: ricarmo@net.em.com.br

The Brazilian Journal of Infectious Diseases 2001;5(5):280-287 (C) 2001 by The Brazilian Journal of Infectious Diseases and Contexto Publishing. All rights reserved.

$1413-8670$
Early neurosyphilis (meningovascular or meningeal) usually occurred within five years after infection whereas late parenchymatous neurosyphilis (tabes dorsalis, general paresis and taboparalysis) occurred five to more than 25 years after infection [9].

HIV infection appears to alter the clinical progression of syphilis, the interpretation of serologic tests of syphilis, and the response to treatment $[10,11]$. The HIV infection increases the risk of rapid progression to early symptomatic neurosyphilis, especially meningitis and meningovascular syphilis, in untreated patients as well as the development of early symptomatic neurosyphilis during the first two years after intramuscular (IM) penicillin treatment $[12,13]$.

The objective of this article is to report two cases of acute syphilitic meningitis in HIV-1infected patients admitted to the Eduardo de Menezes Hospital (a referral hospital for infectious diseases in Belo Horizonte, Brazil) in the year 2000, and to discuss its importance in the differential diagnosis of HIV-infected patients presenting with acute meningeal syndrome. 


\section{Case Report 1}

A 30-year-old black male known to have asymptomatic HIV seropositivity for 2 years without any specific treatment, complained of 3 months of moderate occipital headache. During the one week prior to admission he presented with worsened headache, blurry vision, visual scotomas, conjunctival injection in the right eye and mild left hemiparesis. He denied fever. He had been bisexually promiscuous without any previous sexually transmitted disease. He had a history of cocaine (inhaled) and marijuana abuse but had been abstinent for six months. On examination he was afebrile and had a generalized noninflammatory micropolyadenopathy, oral thrush and perianal warts suggestive of condyloma. His neck was stiff. On eye examination there was evidence of posterior uveitis (iridocyclitis) in the right eye, and the left eye was normal. Neurological examination revealed normal mental status, and lower extremity motor, reflex and sensory function. He had a left facial palsy and hypotonic proximal right upper extremity weakness (MRC grade 4+/5). Laboratory data revealed normal urinalysis, coagulation tests and white blood cell (WBC) count and differential; a mild anemia; and normal electrolytes, creatinine, glucose, calcium, phosphorus, magnesium, and liver function tests. Serum Venereal Disease Research Laboratory (VDRL) test was reactive at a titer of 1:512. Thoracic radiographs were normal and the tuberculin skin test was negative. Cranial computed tomographic scan (CT) showed a hypodense, lacunar lesion in the subcortical region of the left parietal region non-enhanced by i.v. contrast. Examination of the cerebrospinal fluid (CSF) revealed mononuclear pleocytosis $\left(114 \mathrm{WBC} / \mathrm{mm}^{3}\right.$ with $90 \%$ of lymphomononuclear cells), an elevated protein level $(275 \mathrm{mg} / \mathrm{dl})$, glucose $(6 \mathrm{mg} / \mathrm{dl})$, a VDRL test titer of 1:64 and negative Gram's stain, acid-fast stain, India ink smear. The patient received intravenous penicillin $\mathrm{G}$ (24 million units per day) for 21 days for neurosyphilis and oral fluconazole (100mg per day) for 10 days for oral candidiasis. Blood and CSF cultures for bacteria, mycobacteria and fungi were negative. Serum Treponema pallidum hemagglutination test (TPHA) confirmed the diagnosis of syphilis. Other serological tests were done for HBV (HBsAg and anti-HBc-total), HCV (EIA-3.0), HTLV-I/II (EIA) and Trypanosoma cruzi (EIA and RIF). Only anti-HBc-total had a positive result. Subsequent immunovirological evaluation revealed an absolute lymphocyte $\mathrm{CD}_{4}$ count of $175 / \mathrm{mm}^{3}, \mathrm{CD}_{8}$ of $996 / \mathrm{mm}^{3}, \mathrm{CD}_{4} / \mathrm{CD}_{8}$ ratio of 0.18 and serum HIV-1 viral load (by PCR) of 4,600 RNA copies $/ \mathrm{ml}(3.67 \mathrm{log})$. The patient responded well to the treatment and completely recovered from the initial symptoms. Another lumbar puncture after the 21-day course of penicillin showed $12 \mathrm{WBC} / \mathrm{mm}^{3}$ of CSF (100\% lymphomononuclear), protein level of $82 \mathrm{mg} /$ $\mathrm{dl}$, a persistently low CSF glucose $(9 \mathrm{mg} / \mathrm{dl})$ and a nonreactive VDRL test. The patient was discharged and referred to further ambulatory evaluation with the specific recommendation to repeat the lumbar puncture, but was lost to follow-up.

\section{Case Report 2}

A 55-year-old white female with epilepsy controlled with phenobarbital and carbamazepine, was admitted to the Eduardo de Menezes Hospital with a 12-month history of anorexia, weakness, severe weight loss (>30kg), depression and a 6-month history of behavior disorders, emotional instability and periods of mental confusion. She had been treated for psychiatric disorders with chlorpromazine, haloperidol and biperiden with no improvement. Fever began two months prior to admission and she presented to the hospital with a 5-day history of severe headache and significant worsening of her mental status. The patient was divorced, with no history of sexual promiscuity, illicit drug use, or of receiving blood derivatives. Physical examination revealed an awake and malnourished patient, dehydrated $(+/ 4+)$, febrile $\left(39.2^{\circ} \mathrm{C}\right)$, with stiff neck, oral thrush and a diffuse erythemato-macular scaling rash on the trunk, upper and lower extremities (including the palms and soles). Neurological examination revealed confused mental status, with normal cranial nerve function and upper and lower extremity motor, reflex and sensory function. 
Table 1. Neurosyphilis: diagnostic laboratory criteria. From the Centers for Disease Control and Prevention (CDC) [31] and Netherlands Society for Dermatology and Venereology (NSDV) [32].

\begin{tabular}{lll}
\hline Diagnosis of Neurosyphilis & CDC & NSDV \\
\hline Prerequisite & - & Positive CSF-TPHA \\
Compatible case & $\begin{array}{l}\text { Pleocytosis (CSF WBC count } \\
\left.>10 / \mathrm{mm}^{3}\right) \text { and CSF protein } \\
\text { elevated }(>0.50 \mathrm{~g} / \mathrm{l})\end{array}$ & $\begin{array}{l}\text { CSF-IgG index }>0.70 \text { and } \\
\text { CSF WBC count }>10 / \mathrm{mm}^{3}\end{array}$ \\
Confirmed Case & Reactive CSF-VDRL test & Reactive CSF-VDRL test \\
\hline
\end{tabular}

CSF: cerebrospinal fluid; TPHA: Treponema pallidum hemagglutination test; WBC: white blood cell; VDRL: Venereal Disease Research Laboratory.

Table 2. Treatment of neurosyphilis as recommended by the World Health Organization (WHO) [40], the Centers for Disease Control and Prevention (CDC) [31], and the Netherlands Society for Dermatology and Venereology (NSDV) [32].

\begin{tabular}{llll}
\hline Neurosyphilis Stage & WHO & CDC & NSDV \\
\hline Symptomatic & PG 12-24 mU/day i.v. & PG 18-24 mU/day i.v. & PG 0.15 um/kg/day \\
& (2-4 mU/4hrs), & $(3-4 \mathrm{mU} / 4 \mathrm{hrs}), 10-14$ & i.v. 15 days. \\
& 14 days.Alternative: & days. Alternative: & \\
& PPG 1.2mU/day i.m. & PPG 2.4 mU/day i.m. & \\
+ probenecid 4x & + probenecid 4x 500mg & \\
& 500mg 10-14 days & 10-14 days. &
\end{tabular}

\begin{tabular}{llll}
$\begin{array}{l}\text { Asymptomatic neurosyphilis } \\
\text { in late latent syphilis or }\end{array}$ & $\begin{array}{l}\text { No specific } \\
\text { recommendation }\end{array}$ & $\begin{array}{l}\text { See symptomatic } \\
\text { neurosyphilis. }\end{array}$ & $\begin{array}{l}\text { See symptomatic } \\
\text { neurosyphilis. }\end{array}$ \\
duration & & IfCSF examination is & IfCSF examination is \\
& & not performed: BPG 3 x & not performed: BPG 3 \\
& & $\begin{array}{l}2.4 \text { mU i.m. CSF } \\
\text { examination mandatory in }\end{array}$ & x 2.4 mU i.m., plus \\
& & CSF examination after \\
& & HIV-infected patients. & 1 yr. \\
\hline
\end{tabular}

PG: penicillin G i.v.; PPG: procaine penicillin G i.m; BPG: benzathine penicillin G i.m. (3x+ days 1, 8 and 15); CSF: cerebrospinal fluid; Late latent syphilis: latent syphilis acquired $>1$ year (CDC, NSDV) or $>2$ years (WHO) previously or of unknown duration (organ involvement excluded). 
Laboratory data showed mild anemia, leukocytosis with neutrophilia and severe hyponatremia (Na: $123 \mathrm{mEq} /$ 1). No abnormalities were found in the coagulation tests, glucose, renal and liver functions, urinalysis and thoracic radiographs. Serum VDRL test was reactive at a titer of 1:128. Cranial CT was normal. The CSF examination revealed mononuclear pleocytosis (50 $\mathrm{WBC} / \mathrm{mm}^{3}$ with $51 \%$ of lymphomononuclear cells and $49 \%$ polymorphonuclear cells), an elevated protein level (141mg/dl), glucose (34mg/dl), a VDRL test titer of 1:8 and negative Gram's stain, acid-fast stain, India ink smear and latex agglutination for S. pneumoniae, H.influenzae and N. meningitidis. Neurosyphilis treatment with penicillin $\mathrm{G}$ (24 million $U$ daily) was begun and continued for 21 days. In addition, oral ketoconazole (200 mg twice daily) was administered for oral candidiasis. There was improvement of the rash, the meningeal signs and of the mental confusion, but the patient remained hypoactive. Blood and CSF cultures were negative for bacteria, mycobacteria and fungi. Serum TPHA test confirmed the syphilis diagnosis and HIV-1 serologic tests (EIA and WesternBlot) confirmed the HIV-1 coinfection. Serological tests for HBV (HBsAg and anti-HBc-total), HCV (EIA3.0), HTLV-I/II (EIA) and Trypanosoma cruzi (EIA and RIF) were negative. Initial immunovirological evaluation revealed an absolute lymphocyte $\mathrm{CD}_{4}$ count of $343 / \mathrm{mm}^{3}, \mathrm{CD}_{8}$ of $981 / \mathrm{mm}^{3}, \mathrm{CD}_{4} / \mathrm{CD}_{8}$ ratio of 0.35 and serum HIV-1 viral load (by PCR) of 1,200 RNA copies $/ \mathrm{ml}(3.08 \mathrm{log})$. Antiretroviral therapy was begun with zidovudine, lamivudine and nevirapine. Neurological and clinical status improved and a new CSF examination after the 21-day course of penicillin showed $12 \mathrm{WBC} / \mathrm{mm}^{3}$ (100\% lymphomononuclear), mild elevated protein level $(61 \mathrm{mg} / \mathrm{dl})$, normal glucose $(37 \mathrm{mg} / \mathrm{dl})$ and a VDRL test titer of $1: 2$. Once again the CSF microbiological tests were negative. The patient was discharged and referred to followup. Three months after the treatment the serum VDRL test was reactive with a titer of 1:16 and the CSF VDRL test was already non-reactive. At this time, her immunological status had improved with a lymphocyte $\mathrm{CD}_{4}$ count of $657 / \mathrm{mm}^{3}, \mathrm{CD}_{8}$ of $1268 /$ $\mathrm{mm}^{3}$ and $\mathrm{CD}_{4} / \mathrm{CD}_{8}$ ratio of 0.52 .

\section{Discussion}

Syphilis is still a major cause of morbidity in most developing countries and in some areas of North America and Europe, particularly Eastern Europe [14]. In 1999, the overall incidence rate of primary and secondary syphilis in the U.S. was 2.5 per 100,000 population, but some cities showed incidence rates as high as 104.2 per 100,000 population [15]. Globally, there are more than 900,000 cases of pregnant women infected with syphilis every year, resulting in 360,000 fetal or perinatal deaths and in the birth of 270,000 infants with serious or permanent impairment [16].

The exact incidence of neurosyphilis is unknown, and it is unclear why only some patients with syphilis develop neurosyphilis. Its estimated frequency depends on the stage at which the CSF is examined, the risk of the population examined, the adequacy of prior treatment with antibiotics, and the criteria used to define neurosyphilis [17].

Asymptomatic neurosyphilis is common during untreated primary and secondary syphilis $[18,19]$ and during latent syphilis in HIV patients [20].

While symptomatic neurosyphilis is most frequent after 2 years of infection, syphilitic meningitis, the earliest manifestation of neurosyphilis, usually occurs during the first two years following infection. In $10 \%$ of cases, it occurs during the rash of secondary syphilis [21]. In our second reported case, the diffuse maculo-papular rash extending to palms and soles was compatible with secondary syphilis. The most common clinical manifestations of meningeal involvement are headache, nausea, vomiting, stiff neck, delirium, cerebral and cranial nerve dysfunction (mainly of cranial nerves VII and VIII) and, less frequently, polyradiculopathy. Merrit and Moore, in 1935 [22], reported fever in less than $50 \%$ and convulsion in $17 \%$ of 80 patients. They also described three clinical syndromes of acute syphilitic meningitis: (1) acute syphilitic hydrocephalus, with headache, nausea, vomiting, stiff neck and choked disks; (2) acute syphilitic meningitis of the vertex with signs and symptoms of meningeal inflammation, as described in the previous group along with focal cerebral phenomenae such as convulsions, behavioral 
disturbances, aphasia and hemiplegia; and (3) acute syphilitic meningitis, basilar type, consists of cranial nerve palsies due to basilar inflammation.

Syphilis and HIV infection are often closely related [4]. A recent literature review including 30 studies on HIV prevalence among patients with primary diagnosis of syphilis had shown a mean coinfection seroprevalence of $15.7 \%$ in the U.S. [23]. In that review, Blocker, et al. highlighted the need to provideHIV testing for every patient diagnosed with syphilis. Several studies have demonstrated that syphilis constitutes an independent risk factor for HIV infection and the risk seems to be a result of a breakdown in the mucocutaneous barrier due to genital ulceration [24, 25]. The incidence of syphilis among HIV-infected individuals is difficult to estimate, butseroprevalence studies have shown evidence of previous exposure to T. pallidum in 3\% to 35\% HIV-seropositive patients [20, 26, 27]. A study conducted by Carmo, et al. [28] in Belo Horizonte, Brazil (1998), using treponemal tests (EIA or FTA-Abs) showed a syphilis seroprevalence of $37.2 \%$ among 650 HIV-1-infected patients.

The concurrent HIV infection may alter the natural history of syphilis by increasing the propensity of the disease to progress to neurosyphilis, decreasing the latency period before the onset of neurosyphilis, increasing the severity of the manifestations of neurosyphilis, or rendering standard therapy for primary and secondary syphilis inadequate [12]. The neurological manifestations of syphilis most frequently described among HIV-infected patients are acute meningitis and meningovascular disease [6]. Ocular syphilis, present in the first case reported in our study, is strongly associated with neurosyphilis and requires the same therapeutic approach, independent of the time elapsed after the primary infection [29]. Apparently, the central nervous system is involved in all early syphilis cases [6] and the incidence of asymptomatic neurosyphilis is probably underestimated. Some studies show CSF abnormalities in $30 \%$ to $40 \%$ of patients with primary or secondary syphilis $[18,21]$. Early symptomatic neurosyphilis, including the meningovascular presentation shown in our first reported case, was always considered a rare presentation, even in the pre-penicillin era.
The differential diagnosis of syphilitic meningitis, especially in the HIV-infected patient, should include other causes of acute lymphomononuclear meningitis such as tuberculosis, cryptococcosis and other systemic mycoses (e.g. histoplasmosis, paracoccidiodomycosis and coccidioidomycosis), protozoan infections (especially toxoplasmosis and trypanosomiasis in endemic areas), opportunistic viral infections (e.g. cytomegalovirus, herpes virus) and HIV itself. Bacterial meningitis in their early stages or after being partially treated and lymphoproliferative disorders (e.g. lymphomas) also constitute important diseases to be considered in the differential diagnosis of lymphomononuclear meningitis.

In the meningovascular presentation of neurosyphilis, the differential diagnosis should include atherosclerosis, cardiac or aneurismatic emboli, hypercoagulability, presence of anti-phospholipid antibody, inflammatory arterial disease resulting from systemic erythematous lupus, amphetamine use and rheumatoid arthritis [30].

Establishing a definitive diagnosis of neurosyphilis is difficult because there is no "gold standard" criteria. CSF examination is indispensable for diagnosis and to follow response to treatment. The CSF opening pressure may be increased in cases of acute syphilitic meningitis. The traditional hallmarks for the diagnosis of neurosyphilis are the CSF-VDRL test, the CSF WBC count and CSF protein levels (Table 1). Classical abnormalities are a reactive CSF-VDRL test, pleocytosis and elevated CSF protein levels. However, normal values of these three parameters have been found in neurosyphilis [19,33-36]. The CSF WBC count is generally abnormal in early meningovascular neurosyphilis, but it is not necessarily abnormal in late parenchymatous neurosyphilis [9]. The CSF IgG index appears to have higher sensitivity than the CSF protein level [37]. CSF glucose is normal but occasionally it may be slightly decreased, especially in HIV coinfected patients [13]. In our first reported case, CSF glucose was extremely low and remained altered even after the antibiotic course. Unfortunately, his loss to follow-up impaired a more comprehensive analysis of the case.

CSF VDRL, despite its high specificity, has a limited implication due to its low sensitivity, especially in 
asymptomatic neurosyphilis $[19,33,34,36]$. Falsepositive CSF VDRL results can occur occasionally [33], although rarely due to blood contamination. The CSF-FTA-Abs test is more sensitive than the CSFVDRL test for the diagnosis of neurosyphilis, but it is also more prone to false-positive results because of blood contamination or to diffusion of serum immunoglobulin into the CSF. The TPHA and the MHA-TP tests have also been used to detect treponemal antibodies in the CSF. They are highly specific but can yield false positive results due to blood contamination [17]. The use of

Radiologic findings in neurosyphilis are nonspecific and include multifocal infarction and multiple white matter lucencies. The increase in meningeal and parenchymal enhancement observed on brain CT in HIV-infected patients with neurosyphilis most likely reflects the increased incidence of syphilitic meningitis in this group [13]. Overall, the use of magnetic resonance imaging (MRI) is better than the $\mathrm{CT}$ because of the better visualization of the white matter. However, CT can be more useful in the diagnosis of potential complication of cerebrovascular disease such as intracerebral hemorrhage [39]. The radiological finding resulting from occlusive vascular disease such as infarction or arteritis, enhancing cortical lesions with or without enhancement of the adjacent meninges, diffuse or extra-axial focal enhancement, and white matter disease, although nonspecific, are important in the differential diagnosis of neurosyphilis [39].

The treatment of neurosyphilis recommended by the World Health Organization (WHO), the Centers for Diseases Control and Prevention (CDC) and the Netherlands Society for Dermatology and Venereology (NSDV) are listed in Table 2. The therapy of choice is i.v. penicillin $\mathrm{G} 12$ to $24 \mathrm{mU}$ per day for 10 to 14 days $[31,40]$. Although procaine penicillin $(2.4 \mathrm{mU}$ daily) associated with oral probenecid $(4 \times 500 \mathrm{mg})$ could be considered as an alternative regimen, none of the intramuscular penicillin treatment regimens consistently produce treponemicidal penicillin concentrations in CSF [41]. An accurate history accompanied by appropriate skin testing and desensitization is indicated with every patient allergic to penicillin. In special cases requiring use of an alternative therapy, oral doxycycline 200 $\mathrm{mg}$ every 12 hours for 4 weeks or i.v. doxycycline are indicated [40]. Ceftriaxone (1g to 2g IM daily, for 10 to 14 days) has been used [42], but treatment failures have been reported, especially in HIVinfected patients [43].

Parameters for the effectiveness of treatment of neurosyphilis are the clinical picture (which generally does not improve in parenchymatous neurosyphilis and may sometimes progress relentlessly despite treatment) and laboratory findings. CSF WBC count should have decreased at 6 months and CSF should be entirely normal by 2 years. If not, retreatment should be considered. Recurrence and treatment failures of symptomatic neurosyphilis after treatment with IV penicillin have been reported in HIV coinfected patients $[10,13,19]$. Furthermore, it is stated that until CSF WBC count is normal, CSF examination should be performed every 6 months, which enables one to monitor for changes in CSF WBC count, while persistent abnormalities are of less importance [31]. Although normalization of CSF pleocytosis within 6 months after treatment was reported in the majority of neurosyphilis patients [10], others mentioned increased CSF abnormalities within 3 to 6 months after treatment [5]. In some cases, the CSF-VDRL reactivity can persist despite adequate treatment $[10,19,33]$.

Finally, we have to consider the new era of highly active antiretroviral therapy (HAART) that has extended the healthy lifespan of patients infected with HIV since 1995/1996. Further studies are required to clarify the impact of this HAART on the natural history, management and treatment of neurosyphilis in the HIVcoinfected population.

The report of these two cases shows the importance of syphilis in the differential diagnosis of patients with acute meningeal syndrome and its intimate relationship with HIV infection. The impact of HIV infection on the clinical course, treatment response and CSF test interpretation of syphilis has been noted. It remains as a real challenge to clinicians in this new century. 


\section{References}

1. Tichonova L., Borisenko K., Ward H., et al. Epidemics of syphilis in the Russian Federation: trends, origins and priorities for control. Lancet 1997;350:210-3.

2. Smacchia C., Parolin A., Di Perri G., et al. Syphilis in prostitutes from Eastern Europe. Lancet 1998;351:572.

3. Musher D.M. Syphilis, neurosyphilis, penicillin, and AIDS. J Infect Dis 1991;163:1201-6.

4. Rompalo A.M., Shepherd M., Lawlor J.P., et al. Definitions of genital ulcer disease and variation risk for prevalent HIV infection. Sex Transm Dis 1997;24:436-42.

5. Wolters E.C. Treatment of neurosyphilis. Clin Neuropharmacol 1987; 10:143-54.

6. Musher D.M., Hamill R.J., Baughn R.E. Effect of human immunodeficiency virus infection on the course of syphilis and on the response to treatment. Ann Int Med 1990;113:872-81.

7. Roberts M.C., Emsley R.A., Jordaan G.P. Screening for syphilis and neurosyphilis in acute psychiatric admissions. S Afr Med J 1992;82:16-8.

8. van Eijk R.V., Wolters E.C., Tutuarima J.A., et al. Effect of early and late syphilis on central nervous system: cerebrospinal fluid changes and neurological deficit. Genitourin Med 1987;63:77-82.

9. van Voorst Vader P.C. Syphilis management and treatment. Sex Transm Dis 1998;16:699-711.

10. Gordon S.M., Eaton M.E., George R., et al. The response of symptomatic neurosyphilis to high-dose intravenous penicillin $\mathrm{G}$ in patients with HIV infection. N Engl J Med 1994;331:1469-73.

11. de Souza M.C., Nitrini R. Effects of human immunodeficiency virus infection on the manifestations of neurosyphilis. Neurology 1997;49:893-4.

12. Johns D.R., Tierney M., Felsenstein D. Alteration in the natural history of neurosyphilis by concurrent infection with the human immunodeficiency virus. N Engl J Med 1987;316:1569-72.

13. Katz D.A., Berger J.R., Duncan R.C. Neurosyphilis. A comparative study of the effects of infection with human immunodeficiency virus. Arch Neurol 1993;50:243-9.

14. Genest D.R., Choi-Hong S.R., Tate J.E., et al. Diagnosis of congenital syphilis form placental examination: comparison of histopathology, Steiner stain and PCR for Treponema pallidum DNA. Human Pathol 1996;27:366-72.

15. CDC (Centers for Disease Control and Prevention). Primary and secondary syphilis - United States, 1999. MMWR Morb Mort Wkly Rep 2001;50:113-7.

16. CDC (Centers for Disease Control and Prevention). Global disease elimination and eradication as public health strategies. MMWR Morb Mort Wkly Rep 1999;48(Suppl):1-216.
17. Cadazid D., Pachner A.R. Neurosyphilis. In: Goynt R.J., Griggs R.C. eds. Baker's Clinical Neurology on CD-ROM. Philadelphia: Lippincott Williams \& Wilkins, 2000.

18. Lukehart S.A., Hook E.W., Hooker A.S., et al. Invasion of the central nervous system by Treponema pallidum: implications for diagnosis and therapy. Ann Intern Med 1988; 109:855-62.

19. Marra C.M., Critchlow C.W., Hook E.W. ${ }^{\text {rd }}$, et al. Cerebrospinal fluid treponemal antibodies in untreated early syphilis. Arch Neurol 1995;52:68-72.

20. Holtom P.D., Larsen R.A., Leal M.E., et al. Prevalence of neurosyphilis in human immunodeficiency virus-infected patients with latent syphilis. Am J Med 1992;93:9-12.

21. Simon, R.P. Neurosyphilis. Arch Neurol 1985;42:606-13.

22. Merritt H.H., Moore M. Acute syphilitic meningitis. Medicine 1935; 14:119-83.

23. Blocker M.E., Levine W.C., St Louis M.E. HIV prevalence in patients with syphilis, United States. Sex Transm Dis 2000;27:53-9.

24. Greenblatt R.M., Lukehart S.A., Plummer F.A., et al. Genital ulceration as a risk factor for human immunodeficiency virus infection. AIDS 1988;2:47-50.

25. Quinn T.C., Cannon R.O., Glasser D., et al. The association of syphilis with risk of human immunodefficiency virus infection in patients attending sexually transmitted disease clinics. Arch Intern Med 1990;150:1297-1302.

26. Brandon W.R., Boulos L.M., Morse A. Determining the prevalence of neurosyphilis in a cohort co-infected with HIV. Int J STD AIDS 1993;4:99-101.

27. Bordon J., Martinez-Vazquez C., Alvarez M., et al. Neurosyphilis in HIV-infected patients. Eur J Clin Microbiol Infect Dis 1995;14:864-869.

28. Carmo R.A., Andrade C.A., Lima A.A., et al. Prevalence of co-infection by HIV-1 and other sexual/parenteral transmissible pathogens in Brazil (abstract 22195). In: Conference Record: $12^{\text {th }}$ International World AIDS Conference (Geneva) Geneva: CONGREX Switzerland, 1998.

29. Browning D.J. Posterior segment manifestations of active ocular syphilis, their response to a neurosyphilis regimen of penicillin therapy, and the influence of human immunodeficiency virus status on response. Ophthalmology 2000;11:2015-23.

30. Harris D.E., Enterline D.S., Tien R.D. Neurosyphilis in patients with AIDS. Neuroimaging Clin of North Am 1997;7:215-21.

31. CDC (Centers for Disease Control and Prevention). 1998 Guidelines for treatment of sexually transmitted diseases. MMWR Morb Mort Wkly Rep 1998;47:1-116.

32. NSDV, Netherlands Societies for Dermatology and Venereology, Obstetrics and Gynaecology, and Medical Microbiology: Sexually Transmitted Diseases Diagnosis and Therapy Guidelines, 1997. 
33. Hart G. Syphilis tests in diagnostic and therapeutic decision making. Ann Int Med 1986;104:368-76.

34. Wolters E.C., Hische E.A.H., Tutuarima J.A. Central nervous system involvement in early and late syphilis: the problem of asymptomatic neurosyphilis. J Neurol Sci 1988;88:229-39.

35. Tomberlin M.G., Holtom P.D., Owens J.L., Larsen R.A. Evaluation of neurosyphilis in HIV-infected individuals. Clin Infect Dis 1994;18:288-94.

36. MacLean S., Luger A. Finding neurosyphilis without the venereal disease research laboratory-test. Sex Transm Dis 1996;23:392-4.

37. Jones H.D., Urquhart N., Mathias R.G. et al. An evaluation of oligoclonal banding and CSF IgG index in the diagnosis of neurosyphilis. Sex Trans Dis 1990;17:75.

38. Luger A.F., Schmidt B.L., Kaulich M. Significance of laboratory findings for diagnosis of neurosyphilis. Int J STD AIDS 2000; 11:224-34.

39. Brightibill T.C., Ihmeidan I.H., Post M.J.D., et al. Neurosyphilis in HIV-positive and HIV-negative patients: neuroimaging findings. Am J Neuroradiol 1995; $16: 703-11$.

40. WHO and UNAIDS: Management of sexually transmitted diseases. WHO/GPA/TEM/94.1 Rev 1, Geneva, 1997.

41. Hook E.W., Marra C.M. Acquired syphilis in adults. N Engl J Med 1992;326:1060-9.

42. Marra C.M., Boutin P., McArthur J.C. et al. A pilot study evaluating ceftriaxone and penicillin $\mathrm{G}$ as treatment agents for neurosyphilis in human immunodeficiency virus-infected individuals. Clin Infet Dis 2000;30:540-4.

43. Dowell M.E., Ross P.G., Musher D.M. et al. Response of latent syphilis or neurosyphilis to ceftriaxone therapy in persons infected with human immunodeficiency virus. Am J Med 1992;93:481-8. 